\title{
DEVELOPMENT AND QUANTITATIVE ANALYSIS OF AN ADAPTIVE SCHEME FOR BLUETOOTH AND WI-FI CO-EXISTENCE
}

Tanim M. Taher, Kunal Rele, Dennis Roberson

Department of Electrical and Computer Engineering

Illinois Institute of Technology, Chicago, IL.

\begin{abstract}
A smart radio algorithm to supplement the Adaptive Frequency Hopping (AFH) scheme used by Bluetooth devices is developed and studied. The investigation focuses on the Media Access Control (MAC) layer of the Bluetooth. Collision detection, and Bluetooth channel hopping information are used to detect when an IEEE 802.11 wireless Access Point (AP) is operating and the AP's Radio Frequency (RF) channel number (1 through 11). The algorithm then adapts the Bluetooth device to avoid the spectral region occupied by the AP. The performance of this scheme is tested via simulation, and compared with the native AFH system employed by Bluetooth devices.
\end{abstract}

\section{INTRODUCTION}

The Industrial, Scientific and Medical (ISM) radio bands have become very attractive for consumer communication standards that include IEEE 802.11 (Wi-Fi), and IEEE 802.15 (Bluetooth) [1]. Bluetooth was developed as a short-range, low power, and frequency-hopped spread spectrum radio link between devices. Comparatively, Wi-Fi provides higher data rates through a wider bandwidth and is a higher power signal. Due to the high power and large bandwidth occupied by Wi-Fi, Bluetooth performance is adversely affected by Wi-Fi interference. In this paper, an Intelligent Adaptive Frequency Hop (IAFH) algorithm is presented for Bluetooth that was designed to specifically identify Wi-Fi interference and mitigate it. The interference mitigation performance achieved was better than the general AFH scheme.

\section{Bluetooth In Presence OF Wi-Fi}

Bluetooth is used for setting up short-range Wireless Personal Area Networks (WPANs). The radio transceiver uses Frequency Hopped Spread Spectrum (FHSS) with 79, $1 \mathrm{MHz}$ channels in the $2.402-2.483 \mathrm{GHz}$ ISM band [1]. Bluetooth devices hop amongst the 79 channels at a rate of 1600 hops per second in a pseudo-random pattern.

Bluetooth and Wi-Fi interfere when in close proximity to each other [2]. Wi-Fi occupies $22 \mathrm{MHz}$ of spectrum. If the Bluetooth hops to any channel within this $22 \mathrm{MHz}$ spectral space, interference can occur and based on the interference power levels, a significant number of Bluetooth data packets may be lost.

Bluetooth specification version 1.2 tries to address the issue of interference by defining an adaptive frequency hopping (AFH) algorithm [3]. This algorithm [4] allows Bluetooth devices to utilize a channel map that marks channels as good or bad and to avoid hopping on to the bad channels. For mapping, the AFH algorithm utilizes channel sensing through received signal strength indication (RSSI) and bit error rate (BER). The AFH algorithm allows Bluetooth to avoid channels occupied by frequency static systems such as Wi-Fi and wireless security cameras. However, this is a general interference avoidance scheme and Bluetooth devices are unable to ascertain the identity of the interference device.

1. This work is partially supported by the National Science Foundation under contract no. NSF-CNS 0520232.
In theory, Bluetooth with $\mathrm{AFH}$ is supposed to avoid the spectrum that is used by the $22 \mathrm{MHz}$ Wi-Fi channel. However, $\mathrm{Wi}-\mathrm{Fi}$ does not transmit continuously. If the Wi-Fi usage duty cycle is low, the channel map generated by the AFH algorithm may settle on to the default state that records all the 79 Bluetooth channels as good. When the Wi-Fi duty cycle increases suddenly, the AFH needs several seconds for the channel map to correctly identify the bad channels where the $22 \mathrm{MHz} \mathrm{Wi}-\mathrm{Fi}$ exists. This is because the AFH records the 22 bad channels only after the Bluetooth has hopped to each of them and collided with Wi-Fi packets at least once in each of the 22 channels. This means that there is a delay before successful interference avoidance is achieved, but this is offset anytime the Wi-Fi duty cycle drops.

We propose an IAFH scheme for Bluetooth that will actually identify the presence of an IEEE 802.11 Wi-Fi network and the particular RF channel used by it. This will permit successful interference avoidance and rapid response even if the Wi-Fi duty cycle changes.

\section{Proposed Intelligent AFH Algorithm}

The task of the IAFH algorithm is to identify the Wi-Fi channel with minimal packet collision information and then to avoid all the 22 bad channels where the Wi-Fi signals exist. The IAFH needs only about 11 collisions between Wi-Fi and Bluetooth packets to identify the interference source, while the AFH needs at least 22 collisions before it can successfully avoid the Wi-Fi. So the IAFH is at least twice faster.

Using the Bluetooth channel map, the IAFH algorithm first identifies the total number of good and bad Bluetooth channels that fall in the eleven $22 \mathrm{MHz}$ spectral regions spanned by each of the 11 Wi-Fi channels. Optimally there will be 22 good Bluetooth channels in a $22 \mathrm{MHz}$ spectral area with Wi-Fi absent. If, however, the Bluetooth channel map shows that the spectral region spanned by the Wi-Fi Channel 5 has six (6) of the bad Bluetooth channels, Channel 6 has eleven (11) of them, and Channel 7 has five (5), then there is a high probability that a Wi-Fi operates in Channel 6. The IAFH will then identify that a Wi-Fi is operating in Channel 6, and it will mark all the 22 adjacent Bluetooth channels spanning the Wi-Fi Channel 6 area as virtual bad channels and avoid them.

A threshold is used for the minimum number of actual bad Bluetooth channels before the IAFH identifies the Wi-Fi and switches to the Wi-Fi channel avoidance mode for Bluetooth's channel hops. In our simulations, this value is 11 as in the example above. If, for example, there were only $10 \mathrm{bad}$ Bluetooth channels in the Wi-Fi Channel 6 region, the IAFH will not identify the presence of Wi-Fi. Once operating in the Wi-Fi avoidance mode, the IAFH refreshes the list of virtual bad channels periodically. In our simulations, the list is refreshed every $250 \mathrm{~ms}$ during which, the Bluetooth intentionally hops to 
the virtual bad channels where the Wi-Fi should exist. If collisions occur, then it means that the Wi-Fi is still operating, and the Bluetooth remains in the Wi-Fi avoidance mode.

The real advantage of the IAFH scheme is that no hardware changes are needed for Bluetooth 1.2 and above devices. This is a MAC based scheme and only a software upgrade is needed for the Bluetooth enabled devices.

\section{SimUlation ENVIRONMENT}

A simulation environment was created in MATLAB ${ }^{\circledR}$ to test Wi-Fi and Bluetooth interaction for three different frequency hopping algorithms: Bluetooth without $\mathrm{AFH}$, with $\mathrm{AFH}$, and with IAFH. The goal of the simulations was to measure the collision rates between Bluetooth and Wi-Fi packets and how they varied with time. The simulations provide spectrogram outputs as shown by Fig. 1 with time (horizontal axis), frequency (vertical axis), and power (shading intensity) information.

For the Bluetooth signal simulation, a pseudo-random frequency generator selected a hopping frequency out of the possible 79 frequencies. Each Bluetooth time slot was designed to be $625 \mu \mathrm{s}$ based on IEEE 802.15 specs. The simulated AFH and IAFH Bluetooth algorithms utilized channel state estimation maps.

The Wi-Fi signal with $22 \mathrm{MHz}$ BW was generated at the desired Wi-Fi channel. The Wi-Fi data packets were produced by a pseudo-random Poisson process with the packet durations and sequences based on the IEEE $802.11 \mathrm{~b}$ standards. Fig. 1 shows Wi-Fi Channel 6 with centre frequency $2.437 \mathrm{GHz}$ and span 22 $\mathrm{MHz}$. Three different Wi-Fi duty cycles were simulated to model high, medium, and low usages for the Wi-Fi network. Fig. 1 shows medium usage Wi-Fi transmission.

A collision detector was used in the simulation that identified collisions, that is, overlap in the time and frequency domains between AP and Bluetooth packets. The collision detector also provided information to the channel estimator utilized in the simulated Bluetooth device.

\section{RESUltS AND COMPARISON}

The simulation generated output plots as depicted by Fig. 2 . These show the number of collisions per $100 \mathrm{~ms}$, and how this changed with time. In Fig. 2, the performances of the 3 schemes are shown with medium usage Wi-Fi. Table 1 presents the average collision rates for all the nine test cases.

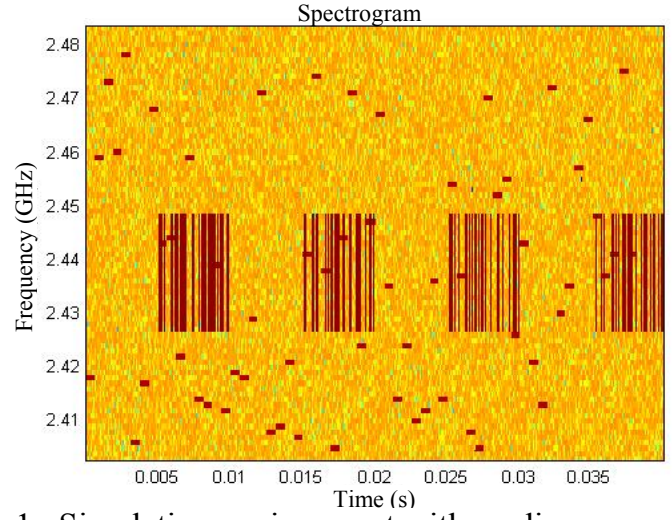

Fig. 1. Simulation environment with medium-usage Wi-Fi

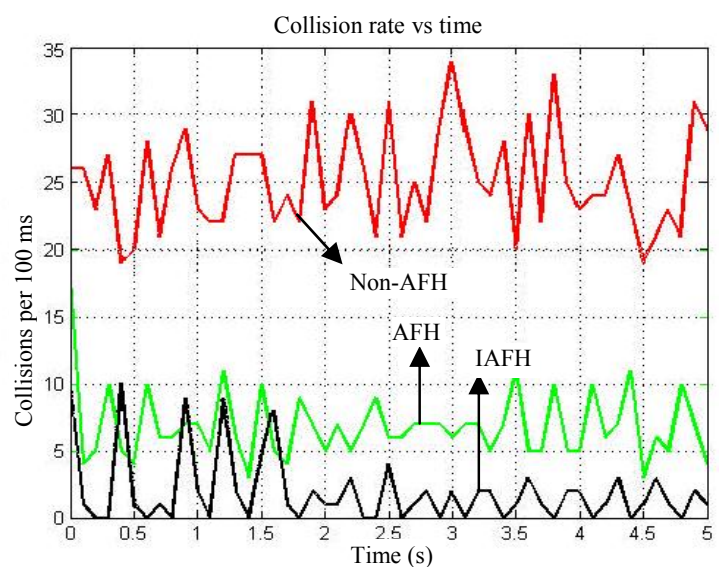

Fig. 2. Collision rates for medium usage $\mathrm{Wi}-\mathrm{Fi}$

Table 1. Average number of collisions per $100 \mathrm{~ms}$.

\begin{tabular}{l|rrr}
\hline \multicolumn{1}{c|}{ Wi-Fi duty } & \multicolumn{3}{|c}{ Bluetooth Algorithms } \\
High & Non-AFH & AFH & IAFH \\
\cline { 2 - 4 } Medium & 45 & 8 & 1.5 \\
Low & 25 & 7 & 1.5 \\
\hline
\end{tabular}

Bluetooth transmits 160 packets in $100 \mathrm{~ms}$. This means that for high and medium-usage Wi-Fi, about $28 \%$ and $16 \%$ of all Bluetooth packets are lost due to Wi-Fi interference, when there is no $\mathrm{AFH}$. For $\mathrm{AFH}$, this number is approximately $5 \%$ for both medium and high Wi-Fi usages, which still is undesirably high. With a low $1 \%$ packet loss rate, the IAFH scheme performs nearly 5 times better than $\mathrm{AFH}$, and between 25 to 45 times better than non-AFH Bluetooth.

For low usage $\mathrm{Wi}-\mathrm{Fi}$, however, the collision rates were similarly all low for non-AFH, AFH and IAFH scenarios. This means that the existing schemes are sufficiently adequate for interference mitigation when the duty cycle of Wi-Fi is low.

\section{Conclusion}

A study was undertaken to examine the co-existence of Bluetooth with Wi-Fi. The deficiencies of simple adaptive frequency hopping in presence of Wi-Fi have been exposed through this study. Hence, an intelligent AFH was designed with the specific goal of detecting Wi-Fi interference and avoiding it. The advantages of IAFH have been demonstrated by the research results.

\section{REFERENCES}

[1] "IEEE Standard for Information technology - 802.15.1: Wireless medium access control (MAC) and physical layer (PHY) specifications for wireless personal area networks (WPANs)," IEEE Std 802.15.1-2005 (Revision of IEEE Std 802.15.1-2002), vol., no., pp. 0_1-580, 2005.

[2] N. Golmie, N. Chevrollier, and O. Rebala, "Bluetooth and WLAN coexistence: challenges and solutions," IEEE Wireless Communications Magazine, Dec 2003.

[3] C. Hodgdon. Adaptive Frequency Hopping for Reduced Interference between Bluetooth and Wireless LAN, 2003. URL: http://www.ericsson.com/bluetooth/files/whitepaper_on_afh_final.pdf.

[4] N. Golmie, O. Rebala, N. Chevrollier, "Bluetooth adaptive frequency hopping and scheduling," Military Communications Conference, 2003. MILCOM 2003. IEEE, vol.2, no., pp. 1138-1142 Vol.2, 13-16 Oct. 2003. 